\title{
Esophageal Motility Disorders: Are We Missing in Our Busy Endoscopy Practice?
}

\author{
Anshuman Elhence $^{1[0} \quad$ Uday C. Ghoshal ${ }^{1}$ \\ ${ }^{1}$ Department of Gastroenterology, Sanjay Gandhi Postgraduate \\ Institute of Medical Sciences, Lucknow, Uttar Pradesh, India \\ J Digest Endosc 2021;12:196-201.
}

\begin{abstract}
Address for correspondence Uday C. Ghoshal, MD, DNB, DM, FACG, RFF, FAMS, FRCP, Department of Gastroenterology, Sanjay Gandhi Postgraduate Institute of Medical Science, Lucknow 226014, Uttar Pradesh, India (e-mail: udayghoshal@gmail.com).
\end{abstract}

\begin{abstract}
\section{Keywords}

- achalasia cardia

- motor dysphagia

- Chicago classification

- hypercontractile esophagus

- distal esophageal spasm

- esophagogastric junction obstruction
\end{abstract}

Esophageal motility disorders (EMDs) form a significant part of a busy endoscopist's practice. Endoscopy plays an all-encompassing role in the diagnosis and management of EMDs including achalasia cardia. The focus on in-vogue third-space endoscopic procedures such as per-oral endoscopic myotomy often digresses the important role of endoscopy. Endoscopic evaluation forms the part of standard first-line evaluation of any dysphagia and serves to rule out a secondary cause such as an esophagogastric junction malignancy and eosinophilic esophagitis. Moreover, endoscopic evaluation may itself provide corroborative evidence that may contribute to the diagnosis of the motility disorder. Achalasia cardia may present with a wide spectrum of endoscopic findings from being entirely normal and the well-known and pathognomonic dilated sigmoid-shaped esophagus with food residue, to lesser-known ornate signs. The evidence on the role of endosonography in EMDs is conflicting and largely restricted to evaluation of pseudoachalasia. High-resolution manometry (HRM) remains the gold standard in the diagnosis of EMDs. Endoscopists must also keep abreast of the latest iteration of the Chicago classification version 4.0 , which differs significantly from its predecessor in being more stringent in making diagnosis of esophagogastric junction outflow obstruction and disorders of peristalsis since these manometric findings may be seen in normal individuals and may be mimicked by opioid use and gastroesophageal reflux. The latest rendition also includes the use of provocative maneuvers and testing in both supine and sitting posture. Despite being the gold standard, there are certain lacunae in the use and interpretation of the Chicago classification of which the users should be well aware. Emerging technologies such as functional lumen imaging probe and planimetry, and timed barium esophagogram fill the lacuna in diagnosis of these motility disorders, which at times is beyond the resolution of HRM.

\section{Introduction}

The busy endoscopy practice of clinicians at academic centers and private setups alike, which had dipped to around less than $10 \%$ of their usual caseload during the coronavirus disease-2019 (COVID-19) pandemic, is now beginning to pick up. ${ }^{1}$ Esophageal motility disorders (EMDs) contribute
DOI https://doi.org/ $10.1055 / \mathrm{s}-0041-1741064$. ISSN 0976-5042. (c) 2022. Society of Gastrointestinal Endoscopy of India. All rights reserved.

This is an open access article published by Thieme under the terms of the Creative Commons Attribution-NonDerivative-NonCommercial-License, permitting copying and reproduction so long as the original work is given appropriate credit. Contents may not be used for commercial purposes, or adapted, remixed, transformed or built upon. (https://creativecommons.org/ licenses/by-nc-nd/4.0/)

Thieme Medical and Scientific Publishers Pvt. Ltd., A-12, 2nd Floor, Sector 2, Noida-201301 UP, India 
a major caseload fraction to the busy endoscopist's practice. Endoscopy has always been an important part of diagnostic work-up and therapeutic armamentarium of EMDs. The role of endoscopy includes, but is no limited to, ruling out alternate etiologies of dysphagia, contributing to the confirmation of the diagnosis and therapy of EMDs. Moreover, some of the EMDs diagnosed with high resolution manometry (HRM), such as distal esophageal spasm (DES) and ineffective esophageal motility (IEM) arise secondary to reflux disease and esophagogastric junction (EGJ) outflow obstruction (EGJOO) may be mimicked by other disorders on HRM, such as opioid-related esophageal pathology; hence, documentation of gastroesophageal junction anatomy and evidence of gastroesophageal reflux on endoscopy is equally important. $^{2,3}$ Most of the endoscopy continuing medical education webinars and conferences for the busy endoscopy practitioner focus on the in-vogue topics of therapeutic endoscopy largely restricted but not limited to per-oral endoscopic myotomy (POEM) and third space endoscopy, but we must not forget the all-encompassing role of endoscopy in the management of EMDs.

As we pull back ourselves to resume our busy endoscopy practice from the inevitable pause due to COVID-19 pandemic, there is time to reflect-are we missing in our busy endoscopy practice the EMDs?

\section{Spectrum and Current Classification of Esophageal Motility Disorders}

The spectrum of EMDs in India has been explored previously. A decade earlier, a study at our center on 250 patients with dysphagia evaluated with conventional manometry showed that achalasia cardia (AC) was the commonest EMD in nearly three-quarters of the patients evaluated over a period of 5 years for dysphagia. ${ }^{4}$ However, with the advent of HRM and emergence of the Chicago classification as the quintessential classification system to classify and interpret EMDs, other EMDs that were earlier not appreciated using conventional manometry are being diagnosed more frequently. A more recent study using HRM has now shown that IEM is the commonest motility disorder accounting for nearly $40 \%$ of patients with dysphagia or retrosternal discomfort and AC is a distant second (26\%). ${ }^{5}$

The most recent iteration of the Chicago classification (version 4.0) was developed with consensus of 52 experts in neurogastroenterology and motility and recommends testing in both supine and upright positions and the use of provocative maneuvers such as multiple rapid swallows in supine position and rapid drink challenge in upright position. ${ }^{6}$ It classifies EMDs into disorders of EGJ outflow (EGJO) and disorders of peristalsis. The disorders of EGJO are characterized by a raised median integrated relaxation pressure (IRP) and are further identified as AC if they are accompanied by absent peristalsis. AC is further subclassified as type I (characterized by $100 \%$ absent peristalsis), type II ( $>20 \%$ swallows showing evidence of panesophageal pressurization in the simultaneous contraction), and type III ( $>20 \%$ swallows showing spasm), with a distal latency (DL)
$<4.5$ seconds indicating simultaneous contractile activity with distal contractile integral $(D C I)>450 \mathrm{~mm} \mathrm{Hg} \mathrm{s} \mathrm{cm}$ (-Fig. 1). The diagnosis of EGJOO needs to be made only when there is manometric evidence of raised IRP in supine and upright positions and $>20 \%$ of the swallows showing intrabolus pressurization along with symptoms of dysphagia and noncardiac chest pain and evidence of obstructive physiology on timed barium swallow (TBE) or functional lumen imaging probe (FLIP). ${ }^{6}$

In the case of normal IRP, disorders of peristalsis are considered. A diagnosis of absent contractility can be made in the presence of $100 \%$ failed peristalsis. The diagnosis of DES requires $>20 \%$ premature swallows with $\mathrm{DL}<4.5$ seconds along with presence of dysphagia and/or noncardiac chest pain. DES differs from achalasia by having some peristalsis ( $\mathrm{DL}>4.5$ seconds), whereas in achalasia all the contractions are simultaneous ( $\mathrm{DL}<4.5$ seconds). Similarly, the diagnosis of hypercontractile esophagus requires $>20 \%$ swallows with $\mathrm{DCI}>8,000 \mathrm{~mm} \mathrm{Hg} \mathrm{s} \mathrm{cm}$ along with symptoms of dysphagia and/or noncardiac chest pain. The diagnosis of IEM has been made more stringent requiring $>70 \%$ ineffective swallows (failed and weak) or $>50 \%$ failed swallows (-Fig. 2).

Since the manometric diagnosis of EMDs can be mimicked by several etiologies such as EGJOO by opioid use and IEM and DES by gastroesophageal reflux disease (GERD), primary EMDs are by themselves thought to be rare. The new iteration emphasizes the fact that a manometric pattern does not equate with a diagnosis of primary EMDs such as EGJOO, DES, and hypercontractile esophagus, and additional symptoms and physiologic tests such as TBE and/or FLIP are required for a conclusive diagnosis. This might increase precision in diagnosis and prevent overtreatment. However, prospective evaluation in the real-world setting remains to be seen.

\section{Identification of Alternate Etiologies Mimicking Esophageal Motility Disorders on Endoscopy}

Pseudoachalasia, by definition, is characterized by an identifiable cause mimicking the clinical and manometric findings of AC. The prevalence of pseudoachalasia varies between 1 and $5 \%$ of all diagnosed cases of $A C{ }^{7}$ The most common cause of pseudoachalasia is an underlying malignancy, most commonly a gastroesophageal junction malignancy (40\%). ${ }^{7}$ However, several benign causes such as amyloidosis and postsurgical alterations can lead to similar clinical and manometric findings. The hypothesis explaining the mechanism of such findings in pseudoachalasia is direct infiltration of the submucosal and muscular plexus by malignancy or paraneoplastic neuropathy arising as a result of distant malignancy. ${ }^{7}$ Clinical clues to a diagnosis of pseudoachalasia include an older age ( $>55$ years), short duration of symptoms ( $<12$ months), and weight loss of $>10 \mathrm{~kg}^{8}$ However, these clinical clues are not specific for pseudoachalasia.

Needless to say, endoscopic evaluation is the first-line test in the evaluation of any dysphagia, but endoscopic evaluation of mucosa is largely normal in pseudoachalasia by 


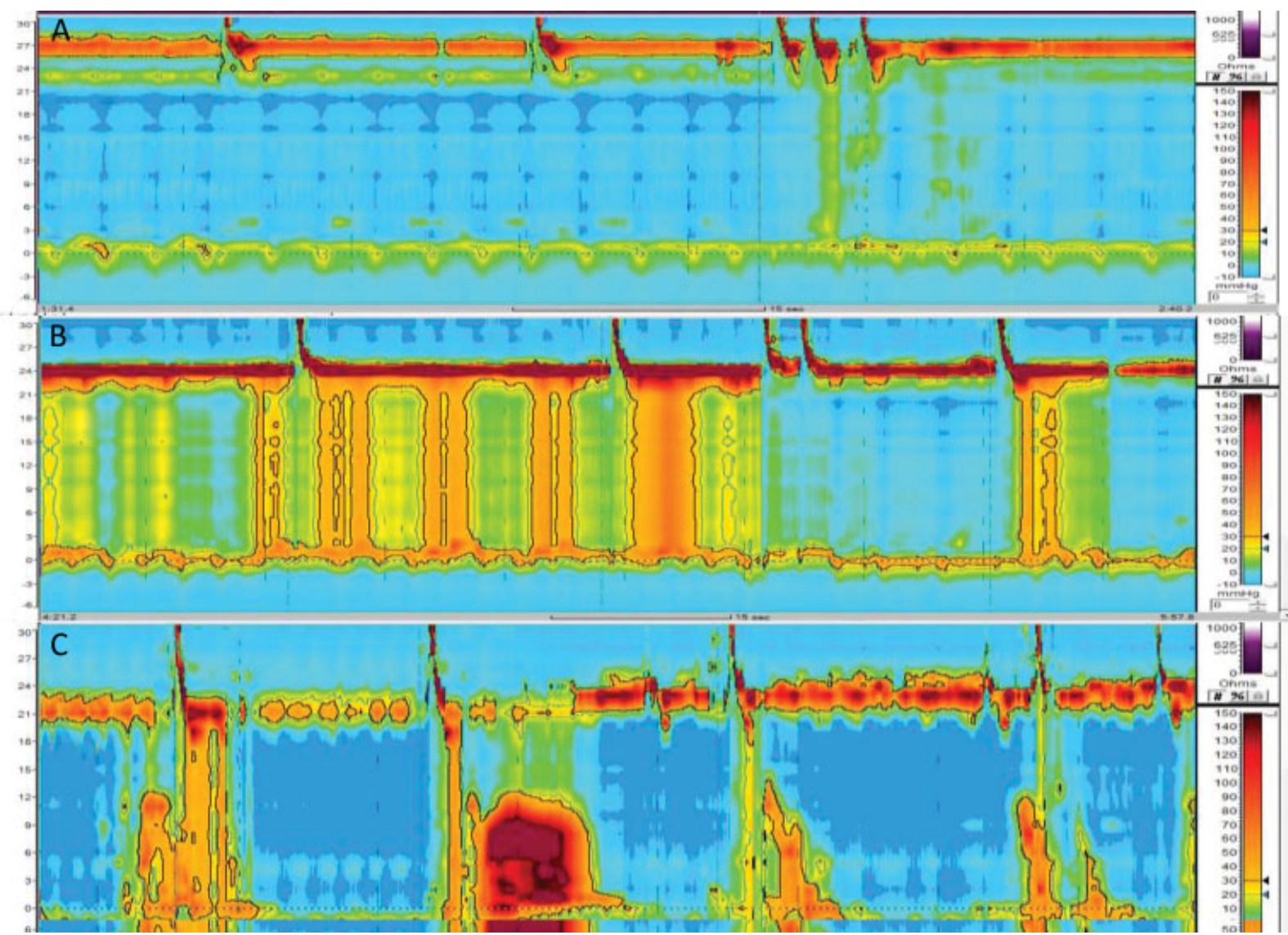

Fig. 1 The high-resolution manometry findings of achalasia cardia with raised integrated relaxation pressure. (A) Type I achalasia with $100 \%$ absent peristalsis. (B) Type II achalasia with more than $20 \%$ swallows showing panesophageal pressurization along with absent peristalsis. (C) Type III achalasia with more than $20 \%$ swallows with spasticity and distal latency less than 4.5 seconds.

definition. Hence, subtle clues that point toward the diagnosis of pseudoachalasia on endoscopy are significant resistance at the EGJ and inability to pass the scope into the stomach. ${ }^{8}$ Also, retroversion of the scope and evaluation of the EGJ in detail to rule out any malignancy is mandatory by the endoscopist. At times, a cross-sectional imaging and endoscopic ultrasound are contributory.

The occurrence of EMDs in association with eosinophilic esophagitis (EoE) is well known. ${ }^{9-11}$ The prevalence of EMDs, including AC, in EoE ranges from 4 to $87 \% .{ }^{9}$ Apart from obvious mechanical strictures and narrowing arising due to mucosal and muscular edema and fibrosis, dysphagia in EoE may arise as a result of underlying motility disturbances in the face of normal endoscopic picture. The release of eosinophilic products that have direct myoirritant and neuroirritant properties is well known. ${ }^{10}$ Hence, it is prudent to have a high clinical suspicion for EoE so that appropriate endoscopic mucosal biopsies may be taken to rule out underlying EoE mimicking these disorders. Recently, we reported a patient with eosinophilic gastroenteritis presenting with AC. ${ }^{12}$

\section{Identification of Specific Esophageal Motility Disorders on Endoscopy}

Most of the EMDs apart from AC do not present with specific endoscopic findings. The spectrum of endoscopic findings in
AC ranges from normal to a grossly dilated sigmoid esophagus. ${ }^{13}$ The mucosa can range from a spectrum of being normal to stained with whitish food and liquid residue or can be entirely not visible due to presence of liquid or solid food residue within the lumen (-Fig. 3). ${ }^{13}$ The mucosa may show changes of stasis esophagitis or secondary candidiasis related to food stagnation. ${ }^{14}$ There may be presence of pulsion diverticula along the length of the esophagus due to the outflow obstruction. The EGJ is characteristically tight but "pops" open on slight pressure. ${ }^{15}$ The nonrelaxing lower esophageal sphincter gives characteristic appearance to the EGJ endoscopically so that the full extent of the palisade vessels is not visible and the mucosa folds in a "rosette"like pattern. ${ }^{16}$ Similarly, in the absence of the characteristic rosette-like pattern, a "gingko leaf"-like pattern of palisade vessels is seen, which is specific for AC. ${ }^{17}$ Other characteristic findings include the pinstripe pattern and the champagne glass appearance on endoscopy. ${ }^{18,19}$ The presence of endoscopically visible but nonspecific spastic and nonoccluding contractions can also provide diagnostic clue toward AC. ${ }^{14}$ Rarely, one may see a simultaneous contraction in patient with achalasia or DES. ${ }^{20}$ However, endoscopy may be entirely normal in patients with EMD, particularly in the early stages.

Although HRM is the gold standard test to diagnose EMDs, the use of endoscopic evaluation as a diagnostic modality has been tested. Cameron et al video-recorded the endoscopic 


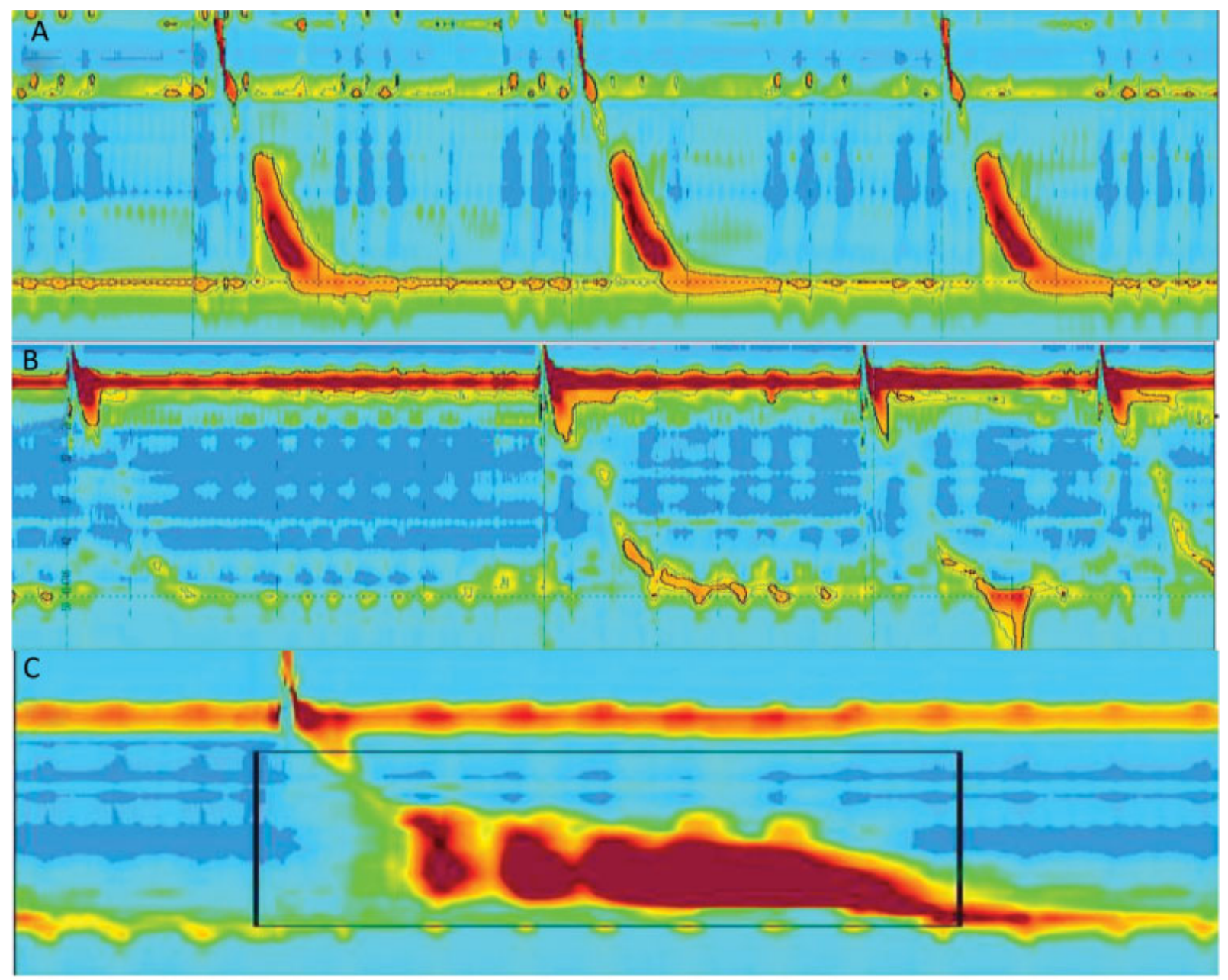

Fig. 2 The high-resolution manometry tracings of (A) normal liquid swallow, (B) ineffective esophageal motility, and (C) hypercontractile esophagus.

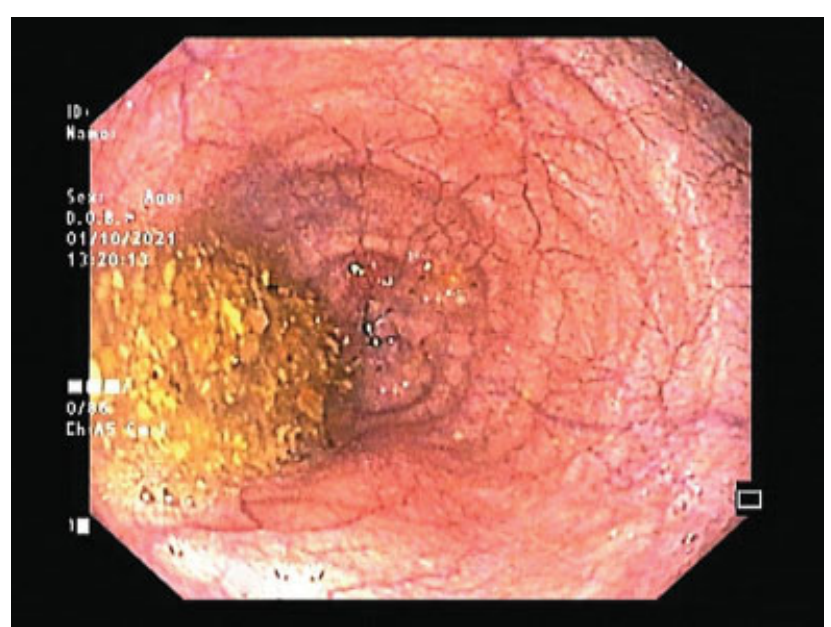

Fig. 3 The characteristic endoscopic findings of achalasia cardia include a dilated esophagus with food residue and secondary mucosal changes related to stasis esophagitis.

evaluation of patients with motility disorders as well as healthy controls. ${ }^{21}$ On evaluation of the recordings by blinded clinicians, the presence of lumen-occluding contractions and no difficulty in opening EGJ was significantly different between healthy controls and those with $\mathrm{AC}{ }^{21}$
However, the small sample size and the low specificity of the findings prevent widespread use of endoscopy as a diagnostic modality. ${ }^{21}$

In another study by Matsubara et al evaluating endoscopic among 380 patients with dysphagia, an abnormal endoscopy was seen in nearly two-thirds of those with an EMD. ${ }^{14}$ They specifically looked for luminal food residue, resistance at EGJ, dilation of the esophageal lumen, and the presence of spastic and non-lumen-occluding contractions by endoscopy. Endoscopy findings were abnormal in 116/127 (91.3\%) of patients with AC, 20/30 (66.6\%) patients with DES, and 6/ 10 (60\%) patients with hypercontractile esophagus but only in $14 / 60(23.3 \%)$ patients with IEM and $1 / 6(16.7 \%)$ patients with fragmented peristalsis. ${ }^{14}$ The utility of these findings as a diagnostic criterion is limited by their low specificity and the effect of drugs used in endoscopy such as benzodiazepines and anticholinergics on the contractility of the esophagus.

\section{Endosonography for Esophageal Motility Disorders}

The role of endoscopic ultrasonography (EUS) has traditionally been limited to rule out pseudoachalasia in patients with 
a high degree of suspicion. The role of EUS in EMDs has been evaluated by Krishnan et al, who in their retrospective case series of 62 patients with EMD showed that EUS was able to contribute to an alternative diagnosis and change the management in $9 / 62(15 \%)$ of the patients. ${ }^{22}$ The patients with a changed diagnosis were predominantly those who has been initially diagnosed as having AC or EGJOO; there was no change in diagnosis or management of those who has been diagnosed with jackhammer esophagus or DES. ${ }^{22}$

Oumrani et al have also evaluated EUS in 69 patients with EMD and showed that esophageal wall thickening was present in $43(62 \%)$ of the patients. ${ }^{23}$ Ultimately, 3/69 (4.3\%) patients were diagnosed as pseudoachalasia but EUS did not contribute to the diagnosis in these cases. ${ }^{23}$ Moreover, they showed that there was no correlation of esophageal thickness with symptoms as assessed by the Eckardt score or with response to treatment. ${ }^{23}$ The esophageal wall thickness was not significantly different among the various types of EMD. ${ }^{23}$ The significance of esophageal muscular wall thickness in $\mathrm{AC}$ remains unresolved with contradictory evidence. $^{24,25}$

\section{Emerging Role of Functional Lumen Imaging Probe and Panometry}

Although HRM is still the gold standard to diagnose EMDs, there are certain cases that cannot be adequately characterized using HRM. The FLIP is a high-resolution impedance planimetry-based device used to assess the distensibility and geometry of the EGJ. It consists of a $240-\mathrm{cm}$-long and 3 -mm-wide catheter on the distal end of which an infinitely compliant $10-\mathrm{cm}$-long balloon is mounted. ${ }^{26}$ The distal end of the catheter that is within the balloon has 16 paired impedance sensors and the distal end of the catheter has a solid-state pressure sensor. The balloon is placed endoscopically or via image assistance across the EGJ after inflating the balloon with a special saline solution. An electrical current is passed between the electrode pairs housed on the catheter within the balloon and the voltage across the impedance electrodes is measured. The impedance between the electrodes is determined by Ohm's law. The calculated impedance is inversely related to the cross-sectional area across the two electrodes, which can be calculated. The diameter of the lumen can also be measured by the same principle. The metric used to assess the distensibility is the distensibility index (DI), which is computed by dividing the lowest median cross-sectional area with the pressure across the distal most pressure sensor. A DI of $2.8 \mathrm{~mm}^{2} / \mathrm{mm} \mathrm{Hg}$ has been suggested as the cut-off for AC. ${ }^{27}$ FLIP can be used to diagnose cases of AC with similar physiology of a nonrelaxing EGJ not meeting the HRM IRP criteria for AC. ${ }^{28}$ Since the HRM catheter measures only lumen-occluding contractions, slight decrease in distensibility or opening of the EGJ is often not appreciable with HRM; however, such cases exhibit a decreased DI and can be diagnosed confidently as AC. Similarly, FLIP has been used to assess the therapeutic response intraoperatively while performing the myotomy during laparoscopic Heller's myotomy and POEM. ${ }^{29}$ The DI has also been used to predict the response to pneumatic dilation, with a $>1.8 \mathrm{~mm}^{2} / \mathrm{mm}$ Hg rise in DI predictive of clinical response. ${ }^{30}$ The Chicago classification version 4.0 recommends using FLIP to confirm the HRM findings of EGJOO since similar manometric findings may be seen in normal individuals and in those with GERD.

The diameters measured by FLIP can be represented on space-time axis with color coding for the diameters in a manner similar to Clouse plots to obtain tracings of contraction pattern throughout the length of the esophagus known as FLIP topography or panometry. These patterns can be used to characterize peristalsis and differentiate between the subtypes of AC. The busy endoscopist must keep abreast of these recent advances in EMD diagnosis and management.

\section{Role of Timed Barium Esophagogram in Diagnosis and Management of EMDs}

A TBE involves serial image acquisition at 1,2, and 5 minutes in left posterior oblique position after ingestion of 100 to $250 \mathrm{~mL}$ of $45 \%$ (weight by volume) of barium sulfate. ${ }^{31}$ In addition to providing anatomical evidence of dilated esophagus and bird's beak appearance typical of achalasia, it provides quantitative measure of esophageal emptying. The height and width of the contrast column can be measured or, alternatively, the area of retained contrast can be measured to give an objective parameter. Usually, the contrast column empties completely at 5 minutes in healthy individuals. Postdilation or myotomy decline in height of column by $50 \%$ is predictor of optimal response. ${ }^{32}$ The advantage of TBE over HRM lies in certain cases in which the manometry catheter cannot be placed across lower esophageal sphincter (LES) and hence IRP cannot be measured. Also, patients with symptoms and HRM findings compatible with EGJOO must have evidence of inadequate emptying or relaxation on physiologic tests such as TBE or FLIP to qualify as EGJOO according to the Chicago version 4.0. FLIP has additional advantage of use as an intraprocedural modality to tailor the length of myotomy but is limited by its availability only at centers of expertise.

\section{Conclusion}

The role of endoscopist in the diagnosis and management of EMDs cannot be undermined. The first-line investigation in patients with dysphagia remains endoscopy. It serves to rule out mechanical obstruction as well as certain etiologies such as pseudoachalasia, GERD, and EoE, which may mimic EMDs on clinical and manometric grounds. Moreover, endoscopy provides supporting role in providing clues for diagnosis of EMDs, primarily AC. However, HRM remains the gold standard for diagnosis of EMDs and their classification. The use of HRM is essential and is the standard of care, as emphasized by the recent Chicago classification version 4.0. The manometric patterns are not diagnostic of primary EMDs such as EGJOO, DES, and hypercontractile esophagus, which require presence of clinical symptoms and additional evidence of deranged emptying and relaxation with TBE and FLIP. Newer 
technologies such as FLIP and panometry are emerging to fill in the gap to diagnose EMDs not adequately characterized by HRM. While endoscopy holds a firm ground in management of EMDs, with procedures ranging from pneumatic balloon dilation, to POEM, to endoscopic botulinum toxin injection, it also holds a firm ground in surveillance and management of postmyotomy reflux that these patients often experience. With a hefty role to play, it is impossible for the busy endoscopist to miss EMDs in their busy practice.

\section{Conflict of Interest}

None declared.

\section{References}

1 Goenka MK, Afzalpurkar S, Ghoshal UC, Guda N, Reddy N. Impact of COVID-19 on gastrointestinal endoscopy practice in India: a cross-sectional study. Endosc Int Open 2020;8(07):E974-E979

2 Yadlapati R, Pandolfino JE, Fox MR, Bredenoord AJ, Kahrilas PJ. What is new in Chicago Classification version 4.0? Neurogastroenterol Motil 2021;33(01):e14053

3 DeLay K, Yadlapati R, Pandolfino JE. Chicago classification of esophageal motility disorders: past, present, and future. Indian J Gastroenterol 2021;40(02):120-130

4 Misra A, Chourasia D, Ghoshal UC. Manometric and symptomatic spectrum of motor dysphagia in a tertiary referral center in northern India. Indian J Gastroenterol 2010;29(01):12-16

5 Goyal O, Bansal M, Sood A. Esophageal motility disorders-symptomatic and manometric spectrum in Punjab, northern India. Indian J Gastroenterol 2017;36(03):202-208

6 Yadlapati R, Kahrilas PJ, Fox MR, et al. Esophageal motility disorders on high-resolution manometry: Chicago classification version 4.0 ${ }^{\circledR}$. Neurogastroenterol Motil 2021;33(01):e14058

7 Barnett DR, Balalis GL, Myers JC, Devitt PG. Diagnosis and treatment of pseudoachalasia: how to catch the mimic [Internet]. Ann Esophagus 2020;3. Accessed August 17, 2021: https://aoe. amegroups.com/article/view/5382

8 Kahrilas PJ, Kishk SM, Helm JF, Dodds WJ, Harig JM, Hogan WJ. Comparison of pseudoachalasia and achalasia. Am J Med 1987;82 (03):439-446

9 Weiss AH, Iorio N, Schey R. Esophageal motility in eosinophilic esophagitis. Rev Gastroenterol Mex 2015;80(03):205-213

10 Spechler SJ, Konda V, Souza R. Can eosinophilic esophagitis cause achalasia and other esophageal motility disorders? Am J Gastroenterol 2018;113(11):1594-1599

11 Moawad F, Lake J, Maydonovitch C, Veerappan G, Bassett J, Wong R. Esophageal motility disorders are uncommon in eosinophilic esophagitis: 39. Am J Gastroenterol 2009;104:S16-S17

12 Mathur A, Mundada K, Verma A, Agrawal V, Verma V, Ghoshal UC. Eosinophilic gastroenteritis presenting as achalasia cardia and responding to botulinum injection and therapy directed against eosinophilic esophagitis. Indian J Gastroenterol 2019;38(06): 554-556

13 Pandolfino JE, Gawron AJ. Achalasia: a systematic review. JAMA 2015;313(18):1841-1852

14 Matsubara M, Manabe N, Ayaki M, et al. Clinical significance of esophagogastroduodenoscopy in patients with esophageal motility disorders. Dig Endosc 2021;33(05):753-760
15 Kuribayashi S, Uraoka T. Can esophageal motility disorders be detected by esophagogastroduodenoscopy? Dig Endosc 2021;33 (05):744-745

16 Iwakiri K, Hoshihara Y, Kawami N, et al. The appearance of rosette-like esophageal folds ("esophageal rosette") in the lower esophagus after a deep inspiration is a characteristic endoscopic finding of primary achalasia. J Gastroenterol 2010;45(04): 422-425

17 Hoshikawa Y, Hoshino S, Kawami N, et al. Possible new endoscopic finding in patients with achalasia: "gingko leaf sign". Esophagus 2020;17(02):208-213

18 Minami H, Isomoto H, Miuma S, et al. New endoscopic indicator of esophageal achalasia: “pinstripe pattern”. PLoS One 2015;10(02): e0101833

19 Gomi K, Inoue H, Ikeda H, et al. New endoscopic classification of the cardiac orifice in esophageal achalasia: champagne glass sign. Dig Endosc 2016;28(06):645-649

20 Kato T, Naiki T, Araki H, Nagaki M, Moriwaki H. Diffuse esophageal spasm. Gastrointest Endosc 2004;60(03):428

21 Cameron AJ, Malcolm A, Prather CM, Phillips SF. Videoendoscopic diagnosis of esophageal motility disorders. Gastrointest Endosc 1999;49(01):62-69

22 Krishnan K, Lin C-Y, Keswani R, Pandolfino JE, Kahrilas PJ, Komanduri S. Endoscopic ultrasound as an adjunctive evaluation in patients with esophageal motor disorders subtyped by highresolution manometry. Neurogastroenterol Motil 2014;26(08): 1172-1178

23 Oumrani S, Barret M, Roseau G, et al. Do we need endoscopic ultrasonography for the workup of patients with esophageal motility disorder? Clin Res Hepatol Gastroenterol 2019;43(05): 608-613

24 Devière J, Dunham F, Rickaert F, Bourgeois N, Cremer M. Endoscopic ultrasonography in achalasia. Gastroenterology 1989;96 (04):1210-1213

25 Ponsot P, Chaussade S, Palazzo L, et al. Endoscopic ultrasonography in achalasia. Gastroenterology 1990;98(01):253

26 Ata-Lawenko RM, Lee YY. Emerging roles of the endolumenal functional lumen imaging probe in gastrointestinal motility disorders. J Neurogastroenterol Motil 2017;23(02):164-170

27 Hirano I, Pandolfino JE, Boeckxstaens GE. Functional lumen imaging probe for the management of esophageal disorders: expert review from the clinical practice updates committee of the AGA institute. Clin Gastroenterol Hepatol 2017;15(03): 325-334

28 Donnan EN, Pandolfino JE. EndoFLIP in the esophagus: assessing sphincter function, wall stiffness, and motility to guide treatment. Gastroenterol Clin North Am 2020;49(03):427-435

29 Campagna RAJ, Carlson DA, Hungness ES, et al. Intraoperative assessment of esophageal motility using FLIP during myotomy for achalasia. Surg Endosc 2020;34(06):2593-2600

$30 \mathrm{Wu}$ PI, Szczesniak MM, Craig PI, et al. Novel intra-procedural distensibility measurement accurately predicts immediate outcome of pneumatic dilatation for idiopathic achalasia. Am J Gastroenterol 2018;113(02):205-212

31 Neyaz Z, Gupta M, Ghoshal UC. How to perform and interpret timed barium esophagogram. J Neurogastroenterol Motil 2013; 19(02):251-256

32 Vaezi MF, Baker ME, Richter JE. Assessment of esophageal emptying post-pneumatic dilation: use of the timed barium esophagram. Am J Gastroenterol 1999;94(07):1802-1807 